\title{
Localizing genes that defer senescence in Drosophila melanogaster
}

\author{
Leo S. Luckinbill,* \\ Joseph L. Graves, ${ }^{*}$ \\ Allen H. Reed $\dagger$ and \\ Sulada Koetsawang*
}

\author{
* Department of Biological Sciences and Institute of \\ Gerontology, Wayne State University, Detroit, \\ Michigan 48202, U.S.A. \\ $\dagger$ Department of Community Medicine, Wayne State \\ University, Detroit, Michigan 48202, U.S.A.
}

Selection for age-specific reproduction has produced replicate stocks in which life span exceeds that in short-lived controls by about 30 per cent, in unpaired individuals. Crosses between a selected long-lived (L) stock, short-lived (S) stock and a strain with balancer chromosomes were used to create all possible combinations of their chromosomes. The longest and shortest-lived genotypes are found to be (LSL) and (SLS), with other combinations distributed between them approximately according to their first and third chromosomes.

Longevity appears to be under polygenic control with contributing elements on all chromosomes. The third chromosome is by far the most influential, accounting for 66 to 72 per cent of the observed variation in females. The first chromosome is less effective. Epistatic interactions are more important in males than females, but are significant only in measurements of single individuals. Some controlling elements for longevity appear to differ in males and females. Crosses of selected stocks with known $P$ and M-cytotype strains show no effect on either sterility or longevity.

\section{INTRODUCTION}

Gerontological studies have resulted in a vast informational base describing aging in a variety of organisms. This has led to the generation of numerous empirically based hypotheses about the genetic and physiological controls on longevity. But the actual fine-scale study of the genetics of aging in Drosophila has awaited the application of selection techniques to establish long- and shortlived stocks from a common ancestral gene pool.

Beginning with Rose and Charlesworth (1981a) and Rose (1984), followed by Luckinbill et al. (1984) and Luckinbill and Clare (1985), studies of selected populations have progressed rapidly, exploring various fundamental aspects of the genetics of longevity using the lines developed. This has included the discovery of additivity in $F_{1}$ populations from crosses to short-lived control stocks as well as a modifying gene-environment interaction (Clare and Luckinbill, 1985; Luckinbill and Clare, 1986) that may have confounded previous studies, and changes in life history characters and physiological parameters correlated with life span (Rose and Charlesworth, $1981 b$; Rose et al., 1984; Service et al., 1985; Service, 1987; Service and Rose, 1985).
Recently, one study has invoked the methods of quantitative genetics in attempting to estimate the number of genetic elements that extend life in selected stocks. Luckinbill et al. (1987) used Lande's (1981) extension of Wright's (1968) reformulation estimating the numbers of effective factors that differentiate two counter-selected parental populations. Measurement of $F_{1}, F_{2}$ and backcrosses were made from crosses of long- with short-lived parental lines. Replicate successive sets of crosses yielded the surprising estimate of a single gene as determining the increased longevity. The estimates of a single factor conflicted, however, with one significant prediction of that unique finding; $F_{2}$ populations from parental lines differentiated by a single gene should exhibit a phenotypic ratio of $1: 2: 1$. Yet no such classes were evident. Thus, though replicate sets of crosses gave strong indication of a single effective factor, at the same time there was some suggestion that a greater number of factors must be involved.

One of the limitations which burden such studies is the extensive assumptions on which the methods used rely. Of these, perhaps the most tenuous of all, in the estimates by Luckinbill et al. (1987), is that genes determining the difference observed between parental lines must have effects 
of equal magnitude and violation of that assumption results in the estimate of the number of factors involved being low.

In this study we use an alternative approach to this problem that both reflects on estimates of gene number and at the same time localizes the elements of interest to specific chromosomes. Here we use nonrecombinant crosses with a balancer stock to create chromosomal substitution lines having all possible combinations of chromosome pairs from long-lived (L) and short-lived (S) control stocks.

\section{METHODS}

The stock $\mathrm{L}_{2} C y O / \operatorname{In}(2 L R) b w^{V I}, d s^{33 K} d p b b w^{V I}$; In $(3 L R) D c x F, D^{3} / S b$ (Bowling Green Stock Center), was used to provide balancer chromosomes in a series of crosses and backcrosses. Stable pericentric inversions incorporate the dominant markers $C y / b w^{\mathrm{VI}}$ and $D^{3} / S b$ on chromosomes II and II respectively. $b w^{\mathrm{VI}}$ is commonly called Plum or $P m$. Crosses of the balancer stock with either the long-lived (LLL) or short-lived (SSS) stocks replaced the first chromosome with paired (L) or (S) homologues to create the stocks:

$\frac{\mathrm{S}}{\mathrm{Y}} \frac{C y}{P m} \frac{D^{3}}{S b} \frac{\mathrm{S}}{\mathrm{S}} \frac{C y}{P m} \frac{D^{3}}{S b}$ and $\frac{\mathrm{L}}{\mathrm{Y}} \frac{C y}{P m} \frac{D^{3}}{S b} \frac{\mathrm{L}}{\mathrm{L}} \frac{C y}{P m} \frac{D^{3}}{S b}$

These base stocks were then again crossed to (LLL) or (SSS) lines to replace chromosomes II and III with those from selected lines. Appendix I shows these. Thus, eight possible substitution combinations were created in which chromosomes I, II and III consisted of paired homologues of either (L) or (S). Lines heterozygous for all chromosomes were constructed by crossing the (SLS) and (LSL) lines. Parental lines (LLL) and (SSS) were reconstructed by backcrossing $F_{1}$ progeny having balancer chromosomes to parental lines. Unreconstructed parental lines were also measured. We attempted to avoid homozygosity as much as possible by crossing large numbers of individuals. Crosses consisted of 50 pairs of individuals raised in vials. For measurement and maintenance of cultures, all lines were reared at high larval density in populations of 50 pair/bottle and were measured for comparison on the third generation after the completion of crosses.

Estimates of longevity here include both adult life span and development. The latter varied by just three days among all the lines described. Longevity was first measured in 30 unpaired males or females in each of eight populations and later in paired populations. Flies were maintained, during measurement, as in Luckinbill and Clare (1985).

Measurements of 960 longevities were analyzed by ANOVA. Variance in longevity of populations showed no particular relationship to means and was heteroscedastic, though not markedly so. Variances were resistant to equalization by conventional transformation methods, but analysis of variance is well known to be little influenced by such inequality when cell numbers are equal, as in this design (Scheffe, 1959; Miller, 1986). Therefore, to show the effects and interactions of chromosomes in the various genotypes, a model-1 (fixed effects) ANOVA was performed on untransformed longevity values using SPSSX (Statistical Package for the Social Sciences) (Nie and Hull, 1979).

Crosses described here mate recently isolated stocks to older marker-bearing stocks that have been in laboratory culture for many years. Experiments were conducted, therefore, to determine whther introduced dysgenic effects may alter longevity in crossed populations. Since the long- and short-lived stocks used here are descended from a population collected from a Michigan orchard in 1979, they are most probably of the P-cytotype. Balancer stocks are likely to be M-cytotype. Crosses between these lines in isolating chromosomes could, therefore, induce considerable levels of mutagenesis and/or male recombination.

To determine the extent to which this has occurred, selected long- and short-lived stocks were reciprocally crossed to both Harwich and Canton$\mathrm{S}$, which are respectively known as $\mathrm{P}$ - and $\mathrm{M}$ cytotype lines. Longevity was measured in populations of 30 unpaired males and females from each cross and fecundity was examined in 100 or more females from each cross, testing for gonodal (GD) sterility.

\section{RESULTS}

Table 1 shows the untransformed mean and standard deviation (s.d.) for both paired and unpaired chromosomal substitution lines. Parental lines shown there and in figs. 1 and 2 are unreconstructed stocks. In females, two reciprocal substitution lines, (SLS) and (LSL), are respectively the shortest and longest lived lines, with the other combinations of the first and third chromosome distributed about evenly between the extremes and parental lines. In fig. 1 the (SLS) combination has a shorter life span than even the short-lived parental line (SSS). 
Table 1 (a) Mean and standard deviation of longevity (in days) for single males and females of chromosome substitution lines. The genotype of lines are shown for major chromosomes I, II and III. (b) Means for genotypes of paired individuals

\begin{tabular}{lll}
\hline & $\begin{array}{l}\text { Average } \\
\text { female } \\
\text { Strain Genotype }\end{array}$ & $\begin{array}{l}\text { Average } \\
\text { longevity s.d. } \\
\text { longevity s.d. }\end{array}$ \\
\hline
\end{tabular}

\begin{tabular}{|c|c|c|c|c|c|}
\hline \multicolumn{6}{|c|}{ (a) } \\
\hline 1 & SSS & $62 \cdot 40$ & 8.93 & $63 \cdot 57$ & 12.66 \\
\hline 2 & SSL & $70 \cdot 77$ & $15 \cdot 24$ & $73 \cdot 53$ & 14.55 \\
\hline 3 & SLS & 54.97 & $15 \cdot 83$ & $65 \cdot 90$ & $16 \cdot 70$ \\
\hline 4 & LSS & 65.67 & $10 \cdot 41$ & $63 \cdot 87$ & $10 \cdot 46$ \\
\hline 5 & LLS & $62 \cdot 77$ & $12 \cdot 29$ & $73 \cdot 23$ & $14 \cdot 49$ \\
\hline 6 & LSL & $74 \cdot 50$ & $4 \cdot 88$ & 78.93 & $11 \cdot 42$ \\
\hline 7 & SLL & 70.07 & $11 \cdot 53$ & $66 \cdot 63$ & 9.90 \\
\hline 8 & LLL & $78 \cdot 90$ & $15 \cdot 26$ & $84 \cdot 29$ & $12 \cdot 94$ \\
\hline \multicolumn{6}{|c|}{ (b) } \\
\hline 1 & SSS & $47 \cdot 13$ & $10 \cdot 49$ & $56 \cdot 33$ & $13 \cdot 64$ \\
\hline 2 & SSL & $60 \cdot 10$ & $18 \cdot 23$ & $65 \cdot 17$ & $15 \cdot 53$ \\
\hline 3 & SLS & $48 \cdot 70$ & $13 \cdot 56$ & $68 \cdot 47$ & 16.59 \\
\hline 4 & LSS & $46 \cdot 23$ & $13 \cdot 49$ & $66 \cdot 40$ & $11 \cdot 38$ \\
\hline 5 & LLS & $55 \cdot 03$ & $14 \cdot 46$ & $68 \cdot 70$ & $15 \cdot 15$ \\
\hline 6 & LSL & $65 \cdot 63$ & $17 \cdot 65$ & $74 \cdot 80$ & $15 \cdot 18$ \\
\hline 7 & SLL & $56 \cdot 00$ & $16 \cdot 15$ & $66 \cdot 20$ & $19 \cdot 61$ \\
\hline 8 & LLL & $78 \cdot 33$ & 16.03 & $79 \cdot 96$ & $11 \cdot 19$ \\
\hline
\end{tabular}

Fig. 2 shows a generally similar pattern with the (SLS) and (LSL) as extremes in males and other combinations distributed between them. One line (LSS) is virtually identical to both the (SSS) and (SLS) lines, but has a slightly shorter average life span than the latter. In males the intermediate isolates are more variable in the position that various combinations exhibit, but a strong effect of the third chromosome is also evident. Among the intermediate genotypes, (SLL) and (LSS), in

\section{FEMALES}

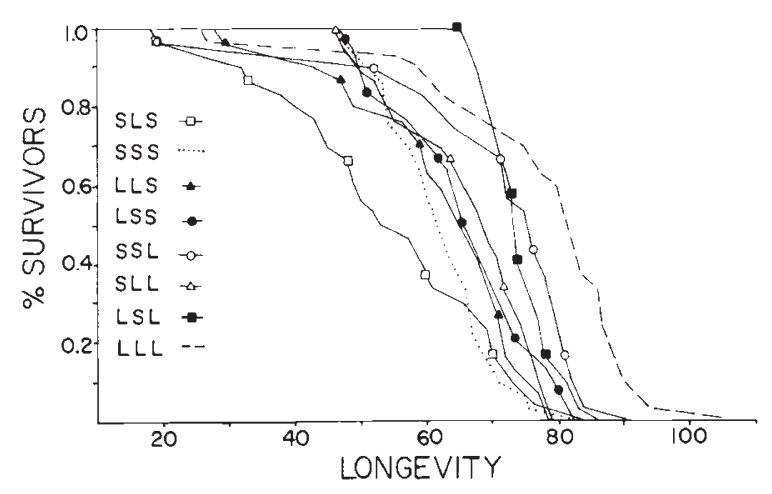

Figure 1 Survival of single females of parental stocks and isogenic chromosomal substitution lines is shown (in days). Dotted lines (....) indicate the short-lived parental stock and dashed lines (- - ) show long-lived parent.

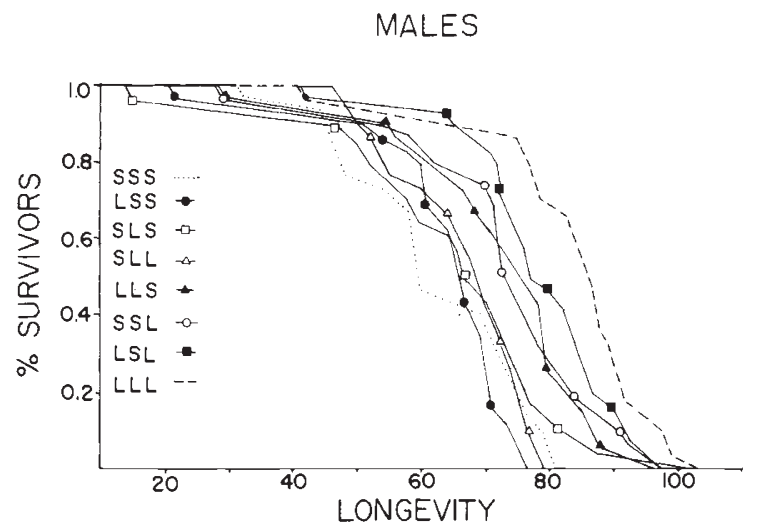

Figure 2 Survival in single males of parental stocks and chromosome substitution isolates is shown.

particular, occupy opposite positions to that expected.

Fig. 3 compares the mean and 95 per cent confidence intervals for single male and female recombinant lines of table 1 . The same trends found in complete survival curves are clear in this comparison; (SLS) and (LSL) are at or near the extremes with other recombinants distributed loosely between according to whether the first and third chromosomes are (L) or (S).

In table 2, heterozygous lines created from reciprocal crosses of the (LSL) and (SLS) substitution lines, show a strong effect in females of one cross. Other means for $F_{1}$ populations are found close to midparent values, as have been most of the previous crosses of parental lines. Reclaimation of parental values in unreconstructed lines by substitution lines is generally excellent. Males and females of the short-lived stock are two to three days lower than values in unreconstructed lines
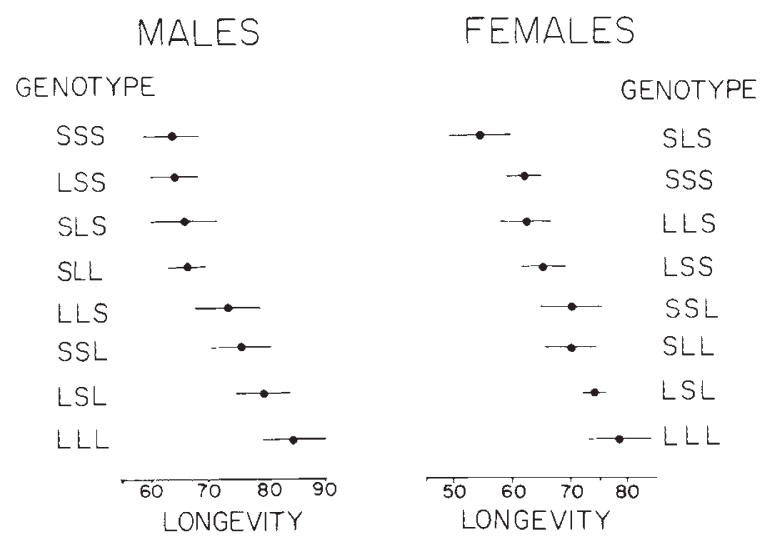

Figure 3 The mean and 95 per cent confidence interval is compared for single males and females of all lines. 
Table 2 Mean and standard deviation of longevity (days) in heterozygotes from crosses of substitution lines SLS with LSL

\begin{tabular}{llllll}
\hline & \multicolumn{2}{l}{ Females } & & \multicolumn{2}{l}{ Males } \\
\cline { 2 - 3 } \cline { 5 - 6 } Cross & Mean & s.d. & & Mean & s.d. \\
\hline $\begin{array}{l}\text { SSS Parental } \\
\begin{array}{l}\text { SLS Males } \\
\times\end{array}\end{array}$ & 60.27 & 5.91 & & 59.60 & 12.23 \\
$\begin{array}{l}\text { LSL Females } \\
\text { LSL Males }\end{array}$ & 71.53 & 11.53 & & & \\
$\times$ & & & & & \\
$\begin{array}{l}\text { SLS Females } \\
\text { LLL Parental }\end{array}$ & 55.33 & 11.15 & & 69.86 & 11.15 \\
& 75.33 & 16.03 & & 80.67 & 17.53 \\
\hline
\end{tabular}

(table 1(a)) and long-lived stocks are within about $3 \cdot 5$ days of their unreconstructed means.

Table 3 shows the comparative contribution of all three chromosomes in the ANOVA of paired and unpaired populations. The variation from the error mean square was less than 2 per cent of the total mean square in all analyses and is excluded from calculations of per cent variation here. In single females, main effects of chromosomes determine more than 91 per cent of the observable variation in life span, while interactions contribute about 9.0 per cent by contrast. Within the main effects, chromosome III accounts for more than 72 per cent of the observed variation alone with

Table 3 The effects and interactions of chromosomes as determined from a model-I (fixed effects) three-way factorial ANOVA of single females or males from substitution and parental lines

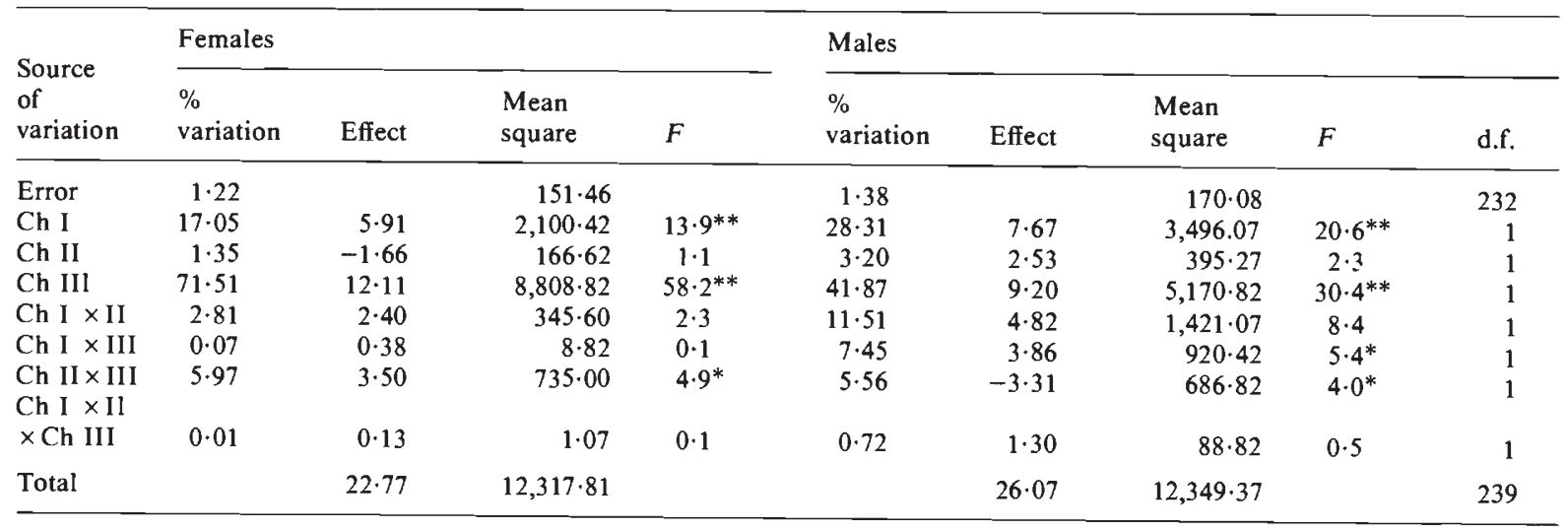

$* 0.01<P<0.05$.

** $P<0.001$.

d.f. $=$ degrees of freedom.

Table 4 Interactions and effects of chromosomes from ANOVAs of paired males and females.

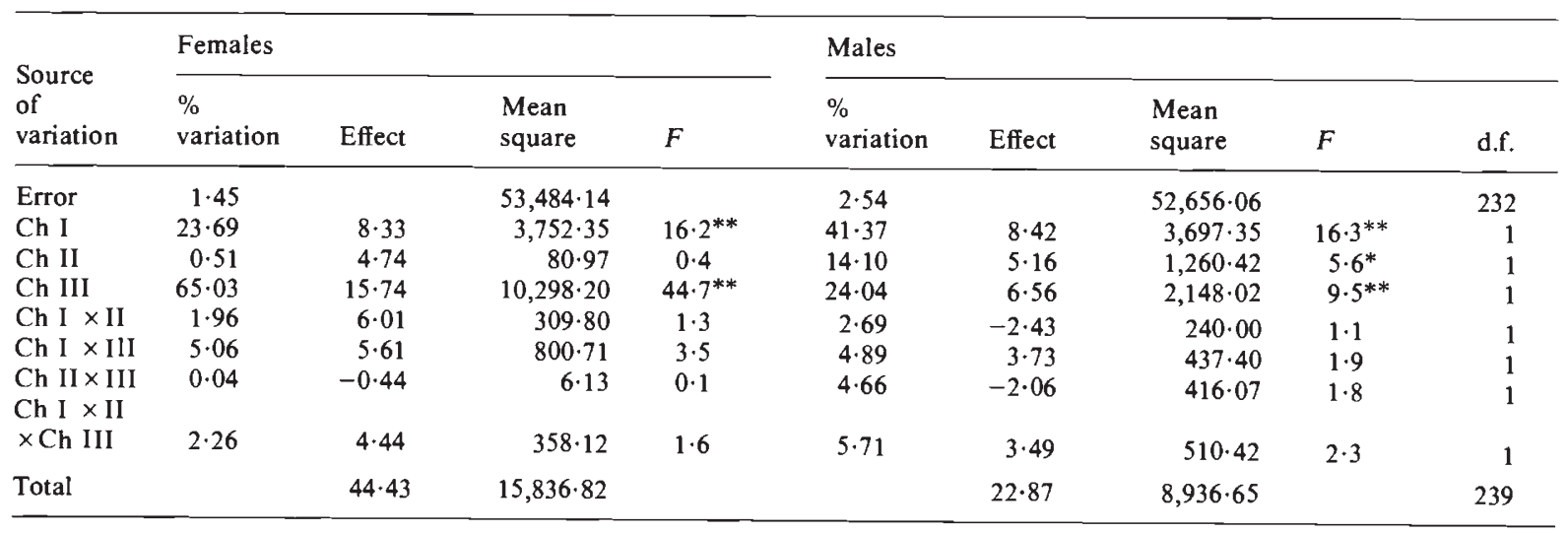

$* 0.01<P<0.05$.

** $P<0.001$.

d.f. $=$ degrees of freedom. 
Table 5 Comparison of per cent sterility and longevity in $F_{1}$ females from crosses of selected stocks to those having an active $P$ or $M$-cytotype

\begin{tabular}{llllr}
\hline Cross & $\begin{array}{l}\text { Cross } \\
\text { type }\end{array}$ & $\begin{array}{l}\text { \% Sterile } \\
\text { females }\end{array}$ & $\begin{array}{l}\text { Mean } \\
\text { longevity }\end{array}$ & s.d. \\
\hline SSS males $\times$ Canton-S females & P & $10 \cdot 1$ & 56.93 & $9 \cdot 97$ \\
Canton-S males $\times$ SSS females & Control & 11.5 & 64.06 & 13.77 \\
LLL males $\times$ Canton-S females & P & $2 \cdot 1$ & 65.60 & 11.98 \\
Canton-S males $\times$ LLL females & Control & 4.0 & 67.03 & 8.97 \\
\hline Harwich males $\times$ SSS feales & M & 2.9 & 76.50 & 8.96 \\
SSS males $\times$ Harwich females & Control & 2.9 & 68.90 & 7.69 \\
Harwich males $\times$ LLL females & M & 6.2 & 73.11 & 10.46 \\
LLL males $\times$ Harwich females & Control & 8.7 & 71.60 & $11 \cdot 75$ \\
\hline
\end{tabular}

the first chromosome also contributing significantly. Independent (main) effects of chromosomes I and III are highly significant $(P<0.001)$ with virtually no effect of the second chromosome. There is, however, a slight but significant interaction between the second and third chromosomes. Heterozygotes were not included in this analysis and the main interactions observed are either additive, as shown by main effects, or epistatic, as shown by first and second order interactions.

The ANOVA of single males in substitution lines shows a somewhat different pattern with respect to first and third chromosomes. The third chromosome also contributes the largest effect there, but it accounts for only 42.5 per cent of the variation observed. Combined main effects contribute about $\frac{3}{4}$ of all variation from the highly significant influence of chromosomes I and III. Chromosome II has little influence here, as in females, but significant two-way (epistatic) interaction occurs between virtually all pairs of chromosomes in males, and account for about 26 per cent of the total variation.

Table 1(b) shows that the same general trends in longevity hold for paired females of substitution lines as for unpaired ones, but with variation. The (LSL) genotype is the longest-lived substitution line, but with (LSS) as well as (SLS) as the shortestlived populations. In paired males the (LSL) genotype is still conspicuous as the most long-lived substitution line, but the means of other stocks are indistinguishably grouped at a value higher than expected.

The ANOVA of longevity in paired lines (Table 4) shows an overall similar pattern of contribution by chromosomes to that in unpaired lines, but with some important differences. In females, the main effects still comprise about 91 per cent of the total with chromosome three providing about 66 per cent of the variation, but the proportional contribution by chromosome one is increased by pairing.
Neither the contribution of the second nor any of the first or second order interactions between chromosomes is significant in paired females or males, though the interactions are again of sizeable combined importance in the latter. In males, the proportional contribution of main effects is increased to 82 per cent but the relative roles of chromosomes I and III become reversed. In paired conditions, chromosome III contributes only about 25 per cent of the total variation in males, while chromosome I increases substantially to about 42.5 per cent. Also, there is a significant contribution by chromosome II found nowhere else.

In table 5, mean and standard deviation (s.d.) of longevity and per cent sterile females are shown for reciprocal crosses between short- and longlived parental stocks of this laboratory with Harwich and Canton-S, respective $\mathrm{P}$ and $\mathrm{M}$-cytoplasmic strains. These crosses indicate the extent to which dysgenic activity can be induced in the parental strains used here or is induced by them in strains of known sensitivity. One of the principal indicators of dysgenic activity is the unequal effects observed in reciprocal crosses, particularly in female sterility. But no especially unbalanced effects are obvious here, either in sterility or longevity. Fig. 4 compares survival in populations of these crosses, which shows no marked reciprocal effects either. Though some dysgenic elements may be activated as a result of crosses, these effects appear generally negligible.

\section{DISCUSSION}

These experiments show that:

(1) The construction of chromosome substitution lines creates stocks in which longevity is distributed across the same range of values as the parental lines from which they were drawn. 

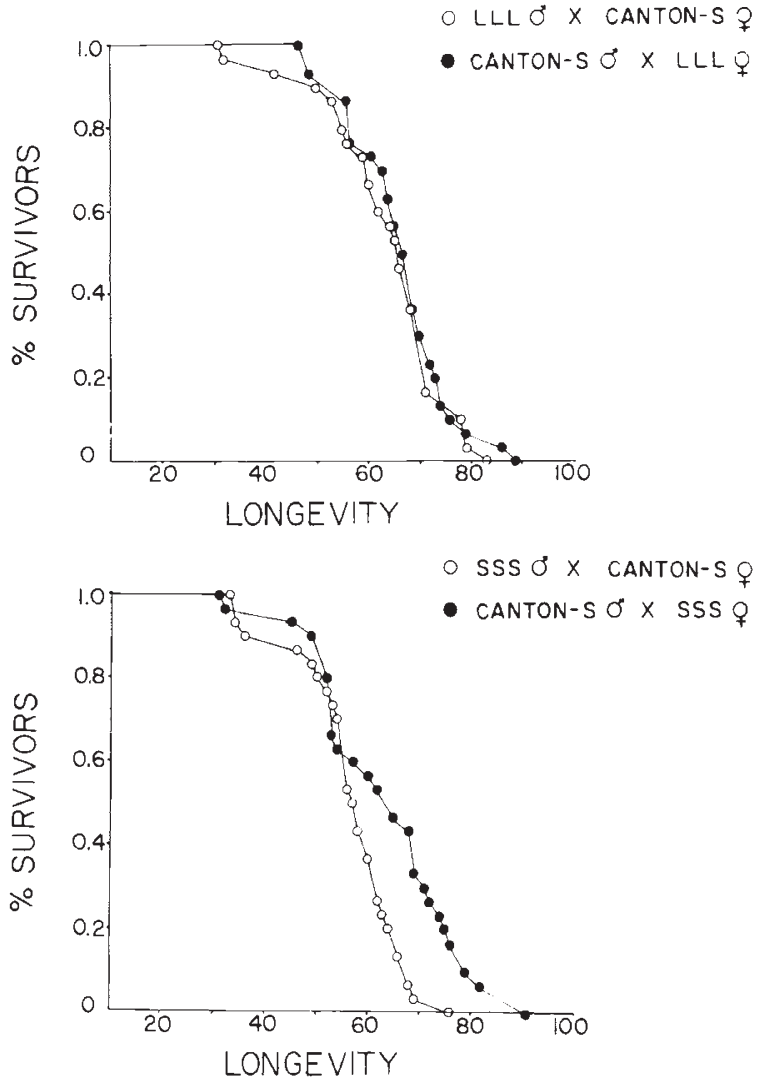

Figure 4 Survival in unpaired $F_{1}$ females from potentially dysgenic crosses (open circles) is compared with that in the nondysgenic reciprocal cross (solid circles).

The respective shortest and longest-lived genotypes are usually SLS and LSL, with other combinations distributed between them. Parental and $F_{1}$ heterozygotes reconstructed from substitution lines reclaim values near those typical for unreconstructed lines.

(2) Control of longevity is definitely polygenic with elements on all three major chromosomes contributing to it disproportionately. The third chromosome is by far the most powerful. Combined effects of the first with third chromosomes account for most of the variation observed, with a slight but significant effect of chrosome two in paired males only.

(3) Genes determining longevity appear to be nonidentical in males and females. Though the same major elements may contribute substantial effects in both, the proportional effects and in some instances genic controls themselves appear to differ. Epistatic interactions are stronger and more numerous in males, but are significantly only in measurements of single individuals.

(4) No dysgenic activity is evident in crosses of selected parental strains used here with stocks of a known P or M-cytotype.

The results of chromosomal substitution experiments, as are reported here require cautious interpretation under the best of circumstances. A composite character such as life span can obviously be greatly disturbed by events such as the insertion of new genes into compatibly selected gene combinations, the generation of new epistatic relationships among chromosomes or other genetic events such as the introduction of homozygosity as can occur in such crosses. In large part, the influence that potential disturbances have in such crosses is shown in the behavior, interpretability and consistency with other indicators in the substitution lines they yield.

The discovery and description of the effects of hybrid dysgenesis make it clear that every analysis involving crosses of recently isolated stocks with older established laboratory populations must consider the potentially catastropic influence of induced mutation and male recombination. Parental stocks used here lack the markers necessary for detecting male recombination. Nevertheless, we have attempted to at least make some estimate of dysgenic effects, as measured by female sterility, and whether longevity and/or survivorship are modified in dysgenic crosses. That no such effects are evident, however, cannot be taken as an indication of the complete absence of dysgenic activity altogether, but merely that, under the limited constraints of these measurements, it appears unimportant. This agrees with and is supported by a more extensive examination of this subject which includes the demonstration that effects on longevity are negligible even where dysgenic influence is strong (Luckinbill, unpublished).

The effects of homozygosity were ostensibly avoided during chromosome substitutions here by crossing large numbers of individuals. Nevertheless, some homozygosity and/or epistatic effects may be in evidence. Longevity in long-lived males and females was a few days lower than the values in unreconstructed parental lines and heterozygous females of one reciprocal cross were unusually low. But the range of values which most such lines assume are comparable to that typical for parental stocks and crosses measured in previous studies.

Measurements of paired females show proportional chromosome effects generally similar to those in single individuals. First and second order interactions between chromosomes are absent in 
all paired lines and the proportional input of the first chromosome is increased by pairing in females and males. In the latter especially, pairing reverses the contribution by chromosomes one and three, with the sex chromosome (one) gaining influence. Chromosome three has by far the greatest influence on longevity.

While this analysis indicates that the genetic basis of longevity is clearly polygenic, the number, effect and distribution of genetic elements remains undetermined. Luckinbill et al. (1987) have made measurements in two extensive sets of crosses that suggest that a single, or at least very few alleles are involved. Yet, that finding of a single factor was not confirmed by predicted phenotypic ratios for $F_{2}$ populations. The results of the substitution analysis here may possibly be of use in interpreting that discrepancy.

Lande's (1981) effective factor estimate assumes an equal influence of the genes measured. Violation of that assumption results in a low estimate of gene number. To the extent that the disproportionate effects of the first and third chromosomes here are representative in this analysis of unequal effects by individual genes, the results of effective factor estimates become more readily understandable. A single, highly influential factor might, if accompanied by a few less effective genes, yield an estimate a single factor, yet give obscured phenotypic ratios in the $F_{2}$. While this may explain the contradictory findings in Luckinbill et al. (1987) the final resolution of that issue rests with the location and functional analysis of the individual genes responsible.

\section{APPENDIX I}

I. $\mathrm{L}_{2} C y O / \operatorname{In}(2 L R) b w^{V I}, d s^{33 K} d p b b w^{V I} ; \operatorname{In}(3 L R)$ $D c x F, D^{3} / S b$ (Bowling Green Stock Center, Bowling Green, Ohio) was used in a series of backcrosses with long-lived (LLL) and short-lived (SSS) selected lines, to replace chromosome I and create the following two marker stocks (Note: $b w^{v I}$ or Plum, as it is commonly called, is symbolized by $P m)$ :

\section{$\frac{\mathrm{S}}{\mathrm{Y}} \frac{C y}{P m} \frac{D^{3}}{S b} \frac{\mathrm{S}}{\mathrm{S}} \frac{C y}{P m} \frac{D^{3}}{S b}$ and $\frac{\mathrm{L}}{\mathrm{Y}} \frac{C y}{P m} \frac{D^{3}}{S b} \frac{\mathrm{L}}{\mathrm{L}} \frac{C y}{P m} \frac{D^{3}}{S b}$}

The following crosses show the creation of three isogenic lines. A total of six substitution lines were created in both LLL and SSS strains by using the reciprocal stocks in the first cross of each series.
II. Chromosome substitution crosses to produce $\underline{S} \underline{L} \underline{L}$

$\overline{\mathrm{S}} \overline{\mathrm{L}} \overline{\mathrm{L}}$

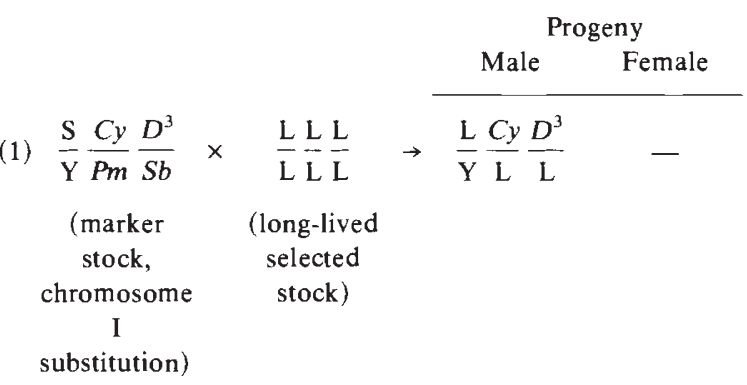

(2) and backcrossing,

$$
\frac{\mathrm{L}}{\mathrm{Y}} \frac{C y}{\mathrm{~L}} \frac{D^{3}}{\mathrm{~L}} \times \frac{\mathrm{S}}{\mathrm{S}} \frac{C y}{P m} \frac{D^{3}}{S b} \rightarrow \frac{\mathrm{S}}{\mathrm{Y}} \frac{C y}{\mathrm{~L}} \frac{D^{3}}{\mathrm{~L}}
$$

(3) and backcrossing again,

$$
\frac{\mathrm{S}}{\mathrm{Y}} \frac{C y}{\mathrm{~L}} \frac{D^{3}}{\mathrm{~L}} \times \frac{\mathrm{S}}{\mathrm{S}} \frac{C y}{P m} \frac{D^{3}}{S b} \rightarrow \frac{\mathrm{S}}{\mathrm{Y}} \frac{C y}{\mathrm{~L}} \frac{D^{3}}{\mathrm{~L}} \quad \frac{\mathrm{S}}{\mathrm{S}} \frac{C y}{\mathrm{~L}} \frac{D^{3}}{\mathrm{~L}}
$$

(4) and crossing from (3),

$$
\frac{\mathrm{S}}{\mathrm{Y}} \frac{C y}{\mathrm{~L}} \frac{D^{3}}{\mathrm{~L}} \times \frac{\mathrm{S}}{\mathrm{S}} \frac{C y}{\mathrm{~L}} \frac{D^{3}}{\mathrm{~L}} \rightarrow \frac{\mathrm{S}}{\mathrm{Y}} \frac{\mathrm{L}}{\mathrm{L}} \frac{\mathrm{L}}{\mathrm{L}} \quad \frac{\mathrm{S}}{\mathrm{S}} \frac{\mathrm{L}}{\mathrm{L}} \frac{\mathrm{L}}{\mathrm{L}}
$$

III. Substituting chromosomes to produce $\frac{\mathrm{S}}{\mathrm{S}} \frac{\mathrm{L}}{\mathrm{L}} \frac{\mathrm{S}}{\mathrm{S}}$

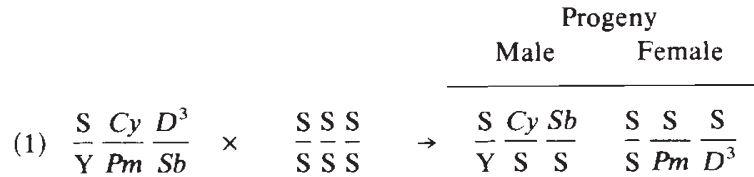

(2) and an $F_{1}$,

$$
\frac{\mathrm{S}}{\mathrm{Y}} \frac{C y}{\mathrm{~S}} \frac{S b}{\mathrm{~S}} \times \frac{\mathrm{S}}{\mathrm{S}} \frac{\mathrm{S}}{\mathrm{Pm}} \frac{\mathrm{S}}{D^{3}} \rightarrow \quad-\quad \frac{\mathrm{S}}{\mathrm{S}} \frac{C y}{P_{m}} \frac{\mathrm{S}}{\mathrm{S}}
$$

(a) and substituting chromosome II from an (L) strain

$$
\frac{\mathrm{S}}{\mathrm{Y}} \frac{C y}{P m} \frac{D^{3}}{S b} \times \frac{\mathrm{L}}{\mathrm{L}} \frac{\mathrm{L}}{\mathrm{L}} \frac{\mathrm{L}}{\mathrm{L}} \rightarrow \frac{\mathrm{L}}{\mathrm{Y}} \frac{C y}{\mathrm{~L}} \frac{S b}{\mathrm{~L}}
$$

(b) and backcrossing,

$$
\frac{\mathrm{L}}{\mathrm{Y}} \frac{C y}{\mathrm{~L}} \frac{S b}{\mathrm{~L}} \times \frac{\mathrm{S}}{\mathrm{S}} \frac{C y}{P m} \frac{D^{3}}{S b} \rightarrow \frac{\mathrm{S}}{\mathrm{Y}} \frac{C y}{\mathrm{~L}} \frac{S b}{\mathrm{~L}}
$$

(c) and backcrossing again to produce females,

$$
\frac{\mathrm{S}}{\mathrm{Y}} \frac{C y}{\mathrm{~L}} \frac{\mathrm{S} b}{\mathrm{~L}} \times \frac{\mathrm{S}}{\mathrm{S}} \frac{C y}{\mathrm{Pm}} \frac{D^{3}}{S b} \rightarrow \quad-\quad \frac{\mathrm{S}}{\mathrm{S}} \frac{\mathrm{L}}{\mathrm{Pm}} \frac{\mathrm{L}}{D^{3}}
$$

(d) and for an $F_{1}$,

$$
\frac{\mathrm{S}}{\mathrm{Y}} \frac{C y}{\mathrm{~L}} \frac{\mathrm{Sb}}{\mathrm{L}} \times \frac{\mathrm{S}}{\mathrm{S}} \frac{\mathrm{L}}{\mathrm{Pm}} \frac{\mathrm{L}}{D^{3}} \rightarrow \frac{\mathrm{S}}{\mathrm{Y}} \frac{\mathrm{L}}{\mathrm{L}} \frac{D^{3}}{S b}
$$


(3) and crossing products,

$$
\frac{\mathrm{S}}{\mathrm{Y}} \frac{\mathrm{L}}{\mathrm{L}} \frac{D^{3}}{S b} \times \frac{\mathrm{S}}{\mathrm{S}} \frac{C y}{P m} \frac{\mathrm{S}}{\mathrm{S}} \rightarrow \frac{\mathrm{S}}{\mathrm{Y}} \frac{\mathrm{L}}{P m} \frac{\mathrm{S}}{D^{3}} \quad \frac{\mathrm{S}}{\mathrm{S}} \frac{C y}{\mathrm{~L}} \frac{D^{3}}{\mathrm{~S}}
$$

(4) and crossing (3)

$$
\frac{\mathrm{S}}{\mathrm{Y}} \frac{\mathrm{L}}{P m} \frac{\mathrm{S}}{D^{3}} \times \frac{\mathrm{S}}{\mathrm{S}} \frac{C y}{\mathrm{~L}} \frac{D^{3}}{\mathrm{~S}} \rightarrow \frac{\mathrm{S}}{\mathrm{Y}} \frac{\mathrm{L}}{\mathrm{L}} \frac{\mathrm{S}}{\mathrm{S}} \quad \frac{\mathrm{S}}{\mathrm{S}} \frac{\mathrm{L}}{\mathrm{L}} \frac{\mathrm{S}}{\mathrm{S}}
$$

IV. Substitution to produce $\frac{\mathrm{S}}{\mathrm{S}} \frac{\mathrm{S}}{\mathrm{S}} \frac{\mathrm{L}}{\mathrm{L}}$

These are reciprocal in many respects with the previous crosses.

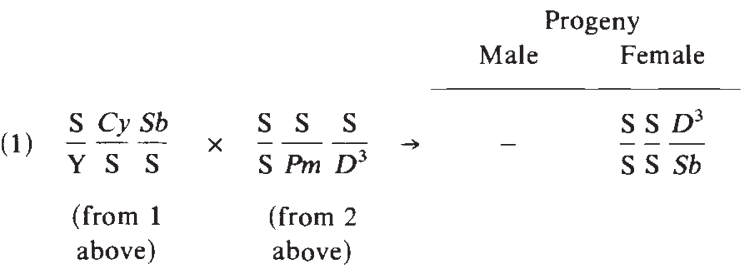

(2) and

$$
\frac{\mathrm{S}}{\mathrm{Y}} \frac{C y}{\mathrm{~L}} \frac{S b}{\mathrm{~L}} \times \frac{\mathrm{S}}{\mathrm{S}} \frac{\mathrm{L}}{P m} \frac{\mathrm{L}}{D^{3}} \rightarrow \frac{\mathrm{S}}{\mathrm{Y}} \frac{C y}{P m} \frac{\mathrm{L}}{\mathrm{L}}
$$

(from $c$

$$
\text { above) }
$$

(3) and crossing,

$$
\begin{array}{ll}
\frac{\mathrm{S}}{\mathrm{Y}} \frac{C y}{P m} \frac{\mathrm{L}}{\mathrm{L}} & \times \frac{\mathrm{S}}{\mathrm{S}} \frac{\mathrm{S}}{\mathrm{S}} \frac{D^{3}}{S b} \\
(\text { from 2) } & (\text { from 1) }
\end{array} \rightarrow \frac{\mathrm{S}}{\mathrm{Y}} \frac{\mathrm{S}}{P m} \frac{\mathrm{L}}{S b} \quad \frac{\mathrm{S}}{\mathrm{S}} \frac{C y}{\mathrm{~S}} \frac{\mathrm{L}}{D^{3}}
$$

(4) and for the $F_{1}$,

$$
\frac{\mathrm{S}}{\mathrm{Y}} \frac{\mathrm{S}}{P m} \frac{\mathrm{L}}{\mathrm{S} b} \times \frac{\mathrm{S}}{\mathrm{S}} \frac{C y}{\mathrm{~S}} \frac{D^{3}}{\mathrm{~L}} \rightarrow \frac{\mathrm{S}}{\mathrm{Y}} \frac{\mathrm{S}}{\mathrm{S}} \frac{\mathrm{L}}{\mathrm{L}} \quad \frac{\mathrm{S}}{\mathrm{S}} \frac{\mathrm{S}}{\mathrm{S}} \frac{\mathrm{L}}{\mathrm{L}}
$$

Acknowledgments J. Graves, S. Koetsawang and W. Krell gave devoted attention to these crosses and experiments. B. Charlesworth and M. Rose offered particularly valuable advice regarding these experiments and their analysis and A. Reed suggested methods for transforming data. Support for this research was provided by grant BSR-8421063 to L.S.L., from the National Science Foundation. The Institute of Gerontology at Wayne State provided a minigrant.

\section{REFERENCES}

CLARE, M. J. AND LUCKINBILL, L. S. 1985. The effects of gene-environment interaction on the expression of longevity. Heredity, 55, 19-29.

LANDE, R. 1981. The minimum number of genes contributing to quantitative variation between and within populations. Genetics, 99, 541-553.

LUCKINBILL, L. S., ARKING, R., CLARE, M. J., CIROCCO, W. C AND BUCK, S. 1984. Selection for delayed sensescence in Drosophila melanogaster. Evolution, 38, 996-1003.

LUCKINBILL, L. S. AND CLARE, M., 1985. Selection for life span in Drosophila melanogaster. Heredity, 55, 9-18.

LUCKINBILL, L. S. AND CLARE, M. 1986. A density threshold for the expression of longevity in Drosophila melanogaster. Heredity, 56, 329-335.

LUCKINBILl, L. S., ClARE, M. J., KRELL, W. L., CIROCCO, W. C. AND RICHARDS, P. A. 1987. Estimating the number of genetic elements that defer senescence in Drosophila. Evolutionary Ecology, 1, 37-46.

MILler, R. G. 1986. Beyond ANOVA, Basics of Applied Statistics. Wiley and Sons, New York, p. 92.

NIE, N. H. AND HULL, C. H. 1979. SPSS: Statistical Package for the Social Sciences. Release 10. McGraw-Hill, New York.

ROSE, M. R. 1984. Laboratory evolution of postponed senescence in Drosophila melanogaster. Evolution, 38(5), 10041010.

ROSE, M. R. AND CHARLESWORTH, B. 1981a. Genetics of life history in Drosophila melanogaster. II. Exploratory selection experiments. Genetics, 97, 187-196.

ROSE, M. R. AND CHARLESWORTH, B. $1981 b$. Genetics of life history in Drosophila melanogaster. I. Sib analysis of adult females. Genetics, 97, 173-186.

ROSE, M. R., DOREY, M. L., COYLE, A. M. AND SERVICE, P. M 1984. The morphology of postponed senescence in Drosophila melanogaster. Can. J. Zool., 62, 1576-1580.

SCHEFFE, H. 1959. The Analysis of Variance. Wiley and Sons, New York, p. 345.

SERVICE, P. M. 1987. Physiological mechanisms of increased stress resistance in Drosophila melanogaster selected for postponed senescence. 1987. Physiol. Zool., 60(3), 321-326.

SERVICE, P. M., HUTCHINSON, E. W., MACKINLEY, M. D. AND ROSE, M. R. 1985. Resistance to environmental stress in Drosophila melanogaster selected for postponed senescence. Physiol. Zool., 58, 380-389.

SERVICE, P. M. AND ROSE, M. R. 1985. Genetic co-variation among life-history components: the effects of novel environments. Evolution, 39, 943-945.

WRIGHT, S. 1968. The Evolution and Genetics of Populations, Vol. I. Genetic and Biometrical Foundations. University of Chicago Press, Chicago. 